\title{
Tubular renal disease-cardiomyopathy syndrome
}

INSERM

\section{Source}

INSERM. (1999). Orphanet: an online rare disease and orphan drug data base. Tubular renal disease-cardiomyopathy syndrome. ORPHA:73224

Tubular renal disease-cardiomyopathy syndrome is characterised by hypokalaemic metabolic alkalosis secondary to a tubulopathy, hypomagnesaemia with hypermagnesuria, severe hypercalciuria and dilated cardiomyopathy. 